\title{
Brecha educativa con las clases virtuales en México durante la pandemia del Covid-19.
}

\section{Education gap with virtual learning in Mexico during Covid-19}

pandemic.

DOI: $10.32870 /$ sincronia.axxv.n80.32b21

\begin{abstract}
Osbaldo Amauri Gallegos de Dios
Centro de Investigaciones y Estudios Superiores en Antropología Social. (MÉXICO)
\end{abstract}

CE: osbaldoamauri27@gmail.com

Esta obra está bajo una Licencia Creative Commons Atribución-NoComercial 4.0 Internacional

Recibido: $16 / 03 / 2021$

Revisado: 22/04/2021

Aprobado: 03/06/2021

\section{RESUMEN}

En el artículo "Brecha educativa con las clases virtuales en México durante la pandemia del Covid-19" se analizan el cierre de escuelas a nivel mundial por la pandemia del coronavirus, los proyectos institucionales de educación virtual a nivel global y local, en universidades de México y España, los retos para los docentes en esta etapa de educación a distancia e híbrida. Por medio de un acercamiento antropológico se analiza la situación sociocultural de la comunidad escolar en México durante el aprendizaje a distancia, lo que permite entender que se debe fortalecer el sistema educativo del Estado para combatir la brecha educativa, el rezago educativo y garantizar el acceso a tecnologías a los alumnos de bajos recursos.

Palabras clave: Covid-19. Educación virtual. Aprendizaje híbrido. Retos. Proyectos institucionales. Rezago educativo. Alumnos de bajos recursos. Brecha educativa. 


\begin{abstract}
In "Education gap with virtual learning in Mexico during Covid-19 pandemic" we analyze the shutting down of schools around the world because of the coronavirus pandemic, the institutional projects of global and local virtual education, in universities of Mexico and Spain, and the teachers' challenges in this period of distance and blended learning. Through an anthropological approach we analyze the school community's sociocultural situation in Mexico during virtual education. Therefore, with this article we will understand that it is necessary to strength the state education system to battle education gap, educational lagging and ensure technology access to low-income students.
\end{abstract}

Key words: Covid-19. Virtual education. Blended learning. Challenges. Institutional projects. Educational lagging. Low-income students. Education gap.

En el 2020, tras la pandemia por el coronavirus, todo el mundo habla de lo importante que es el internet en la sociedad contemporánea. En Estados Unidos, China, Francia o México se pueden realizar una gran cantidad de actividades sin salir de casa: trabajo a distancia, compras y ventas a distancia, relaciones virtuales, juegos virtuales, redes sociales, realidad virtual o educación virtual, entre otras actividades.

El coronavirus ha dejado al descubierto que se puede vivir en la ultramodernidad y realizar todas las actividades necesarias sin salir de casa. Los niños pueden recibir educación virtual y las escuelas, debido a la necesidad de no impartir clases presenciales para no propagar el coronavirus, se han visto obligadas a impartir clases virtuales. Existen programas de educación a distancia que han sido lanzados por la UNESCO y la UNICEF y en el caso de Guadalajara, Jalisco, también se pueden utilizar las iniciativas de educación virtual lanzadas por la SEP y la SEJ. Sin embargo, en un país con grandes carencias económicas como México, ¿es posible la educación virtual para toda la población durante y tras la pandemia provocada por el Covid-19? ¿̇o esta etapa va a incrementar la brecha socioeducativa? 


\section{Escuelas cerradas a nivel mundial por la pandemia del Covid-19}

Para contener la pandemia del Covid-19 una de las acciones mundiales más relevantes fue cerrar todas las escuelas y los especialistas afirman que los paradigmas de la educación van a cambiar. En abril del 2020, la UNESCO señaló que más de mil millones de estudiantes y jóvenes de todo el mundo están afectados por el cierre de escuelas y universidades debido al brote de Covid-19. Por lo tanto, presentó la Coalición Mundial para la Educación Covid-19 que ofrece opciones de aprendizaje inclusivo. Los miembros de esta coalición son: agencias de la ONU, el sector privado, la sociedad civil, organizaciones sin fines de lucro, medios de comunicación asociados, redes y asociaciones. Este proyecto busca: 1) contar con el mayor número de actores y recursos para dar una respuesta efectiva y unificada, 2) coordinar las medidas para llegar a los más desfavorecidos, 3) conciliar las necesidades nacionales y las soluciones mundiales para lograr soluciones eficaces e inmediatas, y 4) proporcionar soluciones tecnológicas, gratuitas, seguras y equitativas a través de la radio, la televisión y las herramientas digitales en línea para las escuelas, estudiantes, maestros y padres (UNESCO, 2020a).

Las universidades a nivel mundial implementarán más clases a distancia a partir de agosto, para evitar la propagación de la pandemia. Silió señala que el ministro de Universidades en España, Manuel Castells, cree que las universidades deben mejorar la enseñanza a distancia, como parte de un "sistema bimodal", ante un posible brote posterior de Covid-19. En España el regreso a clases presenciales será hasta septiembre, por regiones y de manera escalonada por lo que la sociedad debe acostumbrarse a un tipo de enseñanza bimodal, con un aumento de la digitalización (Silió, 2020). La escuela como institución debe mejorar, como afirma el psicopedagogo italiano Francesco Tonnucci, quien considera que la cuarentena para prevenir la propagación del Covid-19 debe ser una oportunidad para mejorar la escuela en diferentes aspectos, uno de ellos, promover el hábito de la lectura (Ayuso, 2020). De esta forma, la educación, en general, va a evolucionar y se utilizarán más las tecnologías. Por consiguiente, los estudiantes mexicanos deberían beneficiarse del conocimiento tecnológico y utilizarlo para recibir una educación de excelencia, tomando en cuenta a los más pobres. 
Uno de los grandes retos en México durante esta pandemia es impartir clases a distancia a más de 34 millones de estudiantes en México porque representan casi un $30 \%$ de la población. Según la información preliminar de la SEP, por medio del Sistema Interactivo de Consulta de Estadística Educativa, la matriculación en el ciclo escolar fue de 34’459,623 estudiantes. 25’253,306 pertenecen a Educación Básica, 5’144,673 a Educación Media Superior (Preparatoria), 3’813,616 a Educación Superior (Licenciatura) y 248,018 a un Posgrado (Programa de las Naciones Unidas para el Desarrollo, 2020, pp. 44-45).

Es importante tomar en cuenta que los estudiantes tienen contacto directo con sus familias, lo que explica que a nivel mundial una de las primeras acciones contra el Covid-19 fue cerrar las escuelas. Para prevenir el contagio, los estudiantes deben recibir las clases desde sus hogares por lo que es esencial tener las estructuras necesarias (tecnología, programa de estudios, planes de trabajo, materiales, trabajo de docentes y directivos, etc.), sin olvidar a la población de bajos recursos que no tiene acceso a la tecnología.

Por su parte, la UNESCO señala que los términos aprendizaje abierto y educación a distancia se refieren a modalidades educativas que apuntan a ampliar el acceso a la educación y a la formación. Todos los países tienen que perfeccionar y renovar los programas de estudios y el conocimiento de los docentes en el uso de las nuevas tecnologías, porque este tipo de aprendizaje juega un papel decisivo en la creación de una sociedad global. El internet tiene un efecto radical en la transformación de la educación, porque los programas más importantes a distancia permiten lograr el mismo número de graduados a un costo menor que las instituciones tradicionales, aunque tienen la desventaja de que muchas comunidades no tienen acceso a las tecnologías. Por estos motivos, la UNESCO continúa apostando al papel fundamental de la educación a distancia en la diversificación de los sistemas educativos (UNESCO, 2002, pp. 10-14).

\section{Proyectos institucionales de educación virtual a nivel global y local}

Tras la pandemia por el coronavirus, en todos los países se habla de lo importante que es la educación virtual porque las escuelas de diferentes niveles, para no propagar el coronavirus, se 
vieron en la necesidad de terminar las clases presenciales. Como respuesta a esta situación, la UNESCO lanzó el proyecto “La educación en América Latina y el Caribe ante el Covid-19” y se divide en cuatro apartados: 1) "Monitoreo de la suspensión de clases" se trata de información de todos los países sobre la suspensión de clases y cuándo planean regresar a las aulas. 2) "Respuestas educativas nacionales" muestra las opciones que ha usado cada país para impartir una educación virtual. 3) "Recursos temáticos" es un esfuerzo del Grupo Regional de Trabajo sobre Educación para América Latina y el Caribe en su respuesta a la crisis Covid-19. 4) "Seminarios Web" son seminarios virtuales (a partir del 17 de abril del 2020) sobre: "Reapertura y funcionamiento seguro de las escuelas", "Recursos y plataformas para la respuesta educativa frente al Covid-19", "Salud en la escuela: preparación para el regreso a clases", entre otros temas (UNESCO, 2020b).

Por su parte, la UNICEF presentó una guía sobre el Covid-19 "Lo que madres, padres, y educadores deben saber" con información sobre cómo prevenir contagios. La perspectiva de la UNICEF en su análisis de los efectos del Covid-19 se enfoca en cómo afecta a los niños y sus familias. Por lo tanto, aborda la educación desde casa, el papel de las madres, el ambiente psicológico, la salud mental de niños y adolescentes, lo que los padres deben saber. Al respecto, en abril lanzó una iniciativa para jugar y aprender con los hijos en tiempos del Covid-19 titulada "\#Aprendo en casa", que se trata de diferentes actividades para aprender de forma virtual (UNICEF, 2020).

La Secretaría de Educación Pública (SEP) tras la pandemia del Covid-19 diseñó tres iniciativas para dar continuidad al ciclo escolar en México durante los meses posteriores a la suspensión de clases. La primera iniciativa fue "Aprende en casa" que consistió en proveer de clases a través de internet y televisión en canales abiertos. La segunda iniciativa fue la "Estrategia de educación a distancia: transformación e innovación para México" que ofrecía herramientas de aprendizaje por medio de Google for Education y Youtube. La última iniciativa fue "Jóvenes en casa" que se enfocaba en ofrecer contenidos de enseñanza cognitiva y emocional a la educación media superior (Programa de las Naciones Unidas para el Desarrollo, 2020, pp. 38). "Aprende en casa" se trata de materiales educativos para aprender a distancia, mediante un sitio web especializado en Educación Básica y existe capacitación en competencias digitales para docentes. Además, los sistemas públicos de 
comunicación transmitirán contenidos educativos y se puede encontrar en: www.sep.gob.mx; www.televisioneducativa.gob.mx; www.aprende.edu.mx y www.telesecundaria.sep.gob.mx (Gobierno de México, 2020).

Finalmente, la Secretaría de Educación Jalisco (SEJ) como parte de las estrategias educativas para contener la pandemia del Covid-19 y la educación a distancia comenzó el proyecto de "Recrea digital" que se trata de una plataforma con información para clases virtuales, en televisión y en la página de Youtube, sobre los recursos didácticos. "Recrea digital" es una plataforma que permite realizar gestiones escolares para que los docentes y alumnos puedan enviar y recibir información (Educación Jalisco, 2020).

\section{Acceso a internet en México y su influencia en la educación virtual}

La educación a distancia no es la única solución, pero debido al gran riesgo de contagio que representa el Covid-19 es la alternativa más segura. En la Ley General de Educación el artículo 84 señala que en Educación Básica se debe enseñar enfocado en las tecnologías y aprendizaje digital por lo que uno de los desafíos el próximo ciclo escolar es usar las tecnologías para la impartición de clases a distancia, tomando en cuenta programa de estudios, planes de trabajo y materiales (Ley General de Educación, 2019). Sin embargo, no se debe olvidar que para poder impartir una educación inclusiva y equitativa se debe tomar en cuenta a la población que no tiene computadora ni internet en sus casas.

Al respecto, Rodolfo Ramírez señala que, en 2015, en México se levantó la Encuesta Nacional sobre Disponibilidad y Uso de Tecnologías de la Información en los Hogares (ENDUTIH) que mostró que, en la población de 6 a 34 años, el 73.6\% tiene acceso al uso de internet por medio de diferentes dispositivos. Por otra parte, en los hogares el 39.2\% contaban con un equipo de cómputo con conexión a internet (Ramírez, 2016).

En el informe "Desarrollo humano y Covid-19 en México: desafíos para una recuperación sostenible" aparece un gráfico sobre el porcentaje de hogares con computadora y conexión a internet por estrato socioeconómico, a partir de los datos del INEGI (ENDUTIH) en 2019. Los 
porcentajes de la conexión a internet son: estrato bajo (16.4\%), estrato medio bajo (40.2\%), estrato medio alto (62\%) y estrato alto (79\%). Este gráfico muestra el gran problema que tienen las familias de escasos recursos económicos para acceder a la educación virtual, porque mientras las estadísticas señalan que alrededor del $56 \%$ de las familias en México cuentan con internet, no dan cuenta de la gran diferencia que existe entre los estratos bajo y alto (Programa de las Naciones Unidas para el Desarrollo, 2020, pp. 39).

Esta situación provoca que en México muchos estudiantes de escasos recursos, de comunidades rurales e indígenas, no tengan las condiciones para seguir las clases o entregar las tareas lo que afecta a los jóvenes de manera desproporcionada. Esta brecha digital también se observa en el cuerpo docente porque un porcentaje considerable no cuenta con capacitación para el uso de las tecnologías en procesos pedagógicos ni los materiales didácticos que corresponde a la enseñanza virtual, por lo que la SEP lanzó la plataforma "Maestras y Maestros en Casa" para que se puedan capacitar (Programa de las Naciones Unidas para el Desarrollo, 2020, pp. 40).

La SEP señala que aún es muy pronto para evaluar los efectos que tendrá el Covid-19 en el aprendizaje (de las y los estudiantes) y en los índices de deserción escolar. Sin embargo, se tiene previsto que impactará de forma importante en la continuidad de los estudios de los estudiantes. Según estimaciones de la SEP, por lo menos 800,000 estudiantes que actualmente cursan el tercer año de secundaria van a interrumpir su educación media superior (preparatoria). Se estima que el próximo ciclo escolar no habrá abandono escolar en el nivel básico en México (preescolar, primaria y secundaria). Sin embargo, $15.55 \%$ de alumnos no continuarán sus estudios en preparatoria y también habrá una notable reducción de los estudiantes universitarios (Programa de las Naciones Unidas para el Desarrollo, 2020, pp. 42-45).

Por lo tanto, las autoridades en México, para hablar de educación básica virtual deben responder ¿cómo se va a impartir educación a distancia a la población que no tiene acceso a internet? o aún más importante ¿cómo se va a impartir educación a distancia a la población de estrato socioeconómico bajo que casi no tiene internet (16.4\%)? Es necesario fortalecer el sistema educativo para cerrar la brecha digital y garantizar el acceso a tecnologías de educación no 
solamente en las escuelas, sino también se deben generar políticas para que los alumnos de bajos recursos pueden tener acceso gratuito al internet y a dispositivos que les permitan continuar con la educación a distancia.

\section{Proyectos de educación a distancia en universidades de México y España}

Un aspecto que en ocasiones resulta complicado es llevar la teoría a la práctica por lo que, en el caso de la educación a distancia, resulta ilustrador el proyecto que realizó en México la Universidad Autónoma Metropolitana (UAM) durante los meses de mayo a junio. El Dr. Eduardo Peñalosa Castro, Rector General de la UAM expuso esta situación en su conferencia: “Papel de las tecnologías en la educación superior en la nueva normalidad: una propuesta" (16 de julio del 2020). Peñalosa mostró la propuesta de educación virtual de la UAM: 1) debe existir un diálogo entre docentes y alumnos, 2) el docente puede recurrir a una variedad de estrategias de enseñanza, 3) el alumno puede aprender autonomía, habilidades del pensamiento y de argumentación, 4) docentes y alumnos tienen un mejor manejo del tiempo, 5) pueden reutilizarse los materiales didácticos para clases posteriores, 6) el diseño educativo puede tener mejor control, 7) existen interacciones sincrónicas (plataformas de videoconferencias como Zoom o Google Meet) y diacrónicas (correos electrónicos) y 8) el alumnado puede reflexionar sobre el uso del internet y aprender a expresarse por medio de las tecnologías.

El objetivo fue ofrecer educación de calidad durante la pandemia y una de las estrategias educativas era que los alumnos podían cursar cualquier clase en el horario que deseaban. Además, era importante que la universidad tomara en cuenta la contingencia provocada por el coronavirus, fuera flexible con los alumnos y los ayudara en el uso de las tecnologías. Así, después de analizar la situación económica de los alumnos, la UAM decidió ofrecer apoyo a la comunidad estudiantil vulnerable y el $13 \%$ de los alumnos (4,633 personas) recibió tablets con chips con internet (Peñalosa, 2020). Por consiguiente, esta experiencia permitió a la UAM valorar la experiencia de educación virtual, conocer las condiciones de la comunidad estudiantil (no todos tienen acceso a una computadora con internet) y darse cuenta de la necesidad de repartir dispositivos con internet para los alumnos de bajos recursos. 
Por otra parte, la Universidad de Guadalajara (UdeG) presentó el Programa de Préstamo de Computadoras "Desde Casa" como una opción para que los estudiantes de bachillerato y licenciatura, del Sistema de Educación Media Superior, UDG Virtual, Centros Universitarios temáticos y regionales de la Red Universitaria, que no tienen un dispositivo o computadora, cursen sus clases en línea y puedan concluir exitosamente el semestre 2020A. En licenciaturas para poder participar se debe consultar el directorio institucional y comunicarse con los centros universitarios para conocer los trámites. En el caso de los estudiantes de bachillerato tienen que registrar su solicitud en su escuela, acudir al plantel con su madre, padre o tutor, llevar los documentos probatorios y el préstamo del equipo será por un máximo de dos días (Desde Casa, 2020).

El rector general de la UdeG, Ricardo Villanueva Lomelí, informó que el 32\% de los estudiantes de esta casa de estudios no tienen computadora, por lo que se lanzó el programa “Desde Casa" con una infraestructura de 500 Ipads y 1000 laptops para prestar a los alumnos, de preparatorias y centros universitarios, de bajos recursos que no cuentan con equipo por lo que podrán seguir estudiando desde sus hogares. De esta forma, se pretende combatir el rezago educativo y generar igualdad de condiciones para los estudiantes (Vázquez, 2020).

En el caso de la Universidad Autónoma de Madrid (UAM), debido a la infraestructura educativa de España, ya existía un programa de préstamo de computadoras para los estudiantes. Sin embargo, debido a la contingencia del coronavirus, el 10 de junio del 2020 fue actualizado para tener un total de 295 computadoras portátiles para el trabajo de los estudiantes dentro y fuera de la universidad. Este equipo se destina únicamente para uso académico. Se trata de un préstamo por un día y no se puede reservar ni renovar. Las computadoras tienen instalado algunos programas informáticos con licencia de uso en la universidad y permiten la conexión a la red inalámbrica de la UAM (Préstamo de portátiles, 2020). De esta forma, puede observarse la importancia de que las escuelas y universidades en México y el extranjero tengan programas de préstamos de computadoras para los alumnos de bajos recursos, lo que permitirá impartir una educación virtual equitativa. 


\section{Retos para los docentes en la educación a distancia}

Tomás Andújar Palma en "La capacitación digital de los docentes, el desafío de la educación que viene" señala que se ha intentado trasladar de la mejor manera posible el aula presencial al aula virtual, pero hay una parte de los docentes que tiene problemas porque no estaban preparados ni tenían las competencias para hacerlo. Además, existen algunos centros educativos que estaban mejor preparados y tenían un proyecto de manejo de tecnologías involucrando a las familias. Por consiguiente, los docentes deben capacitarse en el uso de las tecnologías y aprendizaje en el ámbito digital para poder enseñar de la mejor manera (Andújar, 2020).

Jimena Campuzano en “Maestros en cuarentena: retos de la educación y las clases en línea” afirma que el coronavirus ha llevado a aprender a alumnos, padres y docentes, porque es necesario adentrarse en el uso de las tecnologías para trasladar el conocimiento de las aulas presenciales a las aulas virtuales. Sin capacitación por parte de las autoridades, los docentes han buscado la mejor manera de aprender a usar las aplicaciones y plataformas educativas. Por su parte, los docentes reconocen que no ha sido fácil este ciclo escolar porque pasar de la educación presencial a la virtual fue un cambio drástico. En el tema de la educación, es necesario buscar que las actividades sean las adecuadas para que los estudiantes, dependiendo del nivel educativo, las realicen con sus padres. Además, la enseñanza a distancia no permite a los docentes saber si realmente se consolidan los aprendizajes esperados porque existe poca interacción. Durante la educación a distancia provocada por el coronavirus, los docentes han tenido diferentes muchos retos como familiarizarse con las plataformas educativas, adecuar sus contenidos para que los alumnos sigan aprendiendo y motivarlos en esta época de pandemia (Campuzano, 2020).

Debido a la contingencia por el coronavirus, la Secretaría de Educación Pública (SEP) lanzó la plataforma "Maestras y Maestros en Casa" para que se puedan capacitar en educación virtual. Esta plataforma pone a disposición de los docentes (nivel bachillerato) una serie de recursos para seguir enseñando desde casa y capacitarse por medio de cursos. Ahí, existe una diversidad de recursos didácticos (videos, biblioteca docente, herramientas, recursos online, etc.) acordes a los aprendizajes esperados y contenidos específicos de los programas de estudios de segundo, cuarto y 
sexto semestre. Esta información se puede utilizar para diseñar estrategias de enseñanza y aprendizaje acordes tanto a los estudiantes como a las circunstancias provocadas por el coronavirus (Maestras y Maestros en Casa, 2020). Por lo tanto, los docentes en México deben buscar la forma de capacitarse en el uso de las tecnologías y utilizar las plataformas educativas para impartir una educación a distancia que motive a los estudiantes.

\section{¿Educación híbrida en el ciclo escolar 2020-2021?}

Desde que empezó la pandemia por el coronavirus, la fecha del regreso a clases presenciales se ha cambiado varias veces en México, aunque un aspecto importante es que no todos los Estados se encuentran en la misma situación. Por ejemplo, en el Estado de Jalisco (con Guadalajara la segunda ciudad más grande del país) y otros nueve Estados, desde el 17 de marzo se suspendieron las clases debido al Covid-19, adelantándose varias semanas al plan nacional, lo que ocasionó cierta confusión al inicio de la pandemia (Expansión política, 2020).

En Jalisco al inicio se pensaba que después de dos meses habría una vuelta a las escuelas. Luego, las autoridades indicaron que en septiembre se iniciaría de manera normal el ciclo escolar. Posteriormente, se señaló que en noviembre. Finalmente, a principios del mes de diciembre del 2020 el gobernador del Estado de Jalisco, Enrique Alfaro, expresó que si en términos de salud hay condiciones los alumnos podrían volver a las clases presenciales el 25 de enero del 2021. Sin embargo, las clases presenciales son optativas y si algunos padres no se sienten seguros no están obligados a enviarlos. Esta situación muestra que, ante la incertidumbre sobre la duración de la pandemia, no existe un plan en el Estado de Jalisco sobre cómo regresar a las clases presenciales. Alfaro expuso que durante la contingencia por el coronavirus han muerto 49 maestros en activo en Jalisco, por lo que aun sin asistir a clases presenciales viven en un ambiente de riesgo. Para él, las clases presenciales no pueden ser sustituidas por una computadora o el internet y el contacto entre maestros y alumnos es parte de la salud emocional y del proceso de aprendizaje (Ramírez, 2020). No obstante, en una situación tan grave como la que se está viviendo a nivel mundial con el Covid-19, es importante resaltar que las familias de bajos recursos son las que se verán más afectadas porque 
en muchos casos han perdido sus trabajos, se han enfermado del coronavirus y no han podido seguir el proceso de clases virtuales.

Por otra parte, luego de conversar con las familias de los estudiantes o el personal que trabaja en Educación Básica, se puede conocer que un porcentaje considerable cree que lo mejor es terminar el ciclo escolar de manera virtual (como se ha realizado desde agosto) por el riesgo que representa el contagio del coronavirus y que puede afectar sobre todo a la población más pobre y vulnerable. Además, a nivel mundial se está viviendo una nueva oleada de contagios por la temporada de invierno.

Un aspecto que sobresale en este plan del Gobernador de Jalisco para regresar a las clases presenciales a finales de enero del 2021 es que en ningún momento ha mencionado algo sobre la educación híbrida o un plan coordinado de educación híbrida a nivel estatal, lo que permitiría volver paso a paso a las clases presenciales. Blended Learning es conocido como aprendizaje híbrido o semi-presencial y se empezó a utilizar desde hace una década. No se trata solamente de usar nuevas tecnologías educativas y combinar las clases virtuales con las presenciales, sino de darle a cada alumno una experiencia personalizada. Por tal razón, en el regreso a las clases, se deben agregar las herramientas digitales a la educación presencial en escuelas públicas y privadas y permitir a los estudiantes elegir sus horarios de estudio. Además, es necesario adaptar los planes de estudio tomando en cuenta la educación presencial y virtual (Servín, 2020).

El aprendizaje híbrido permite integrar los avances tecnológicos del aprendizaje virtual con la interacción de las clases presenciales, como lo indican François Bédard, Patrick Pelletier y Christiane Le Clech en "L’apprentissage hybride". El aprendizaje híbrido permite al maestro experimentar un acercamiento centrado en los estudiantes y sus preferencias de aprendizaje, por lo que ofrece más flexibilidad a los estudiantes que deben combinar estudios-trabajo-familia (Bédard, Pelletier y Le Clech, 2020).

Por su parte, el 22 de julio del 2020, es decir cuatro meses antes, Esteban Moctezuma Barragán, Secretario de Educación Pública en México, encabezó la XLIX Reunión Ordinaria virtual del Consejo Nacional de Autoridades Educativas (CONAEDU) donde expresó que el enfoque pedagógico 
que existirá en el futuro será híbrido: con educación virtual y presencial, conforme a las necesidades de cada sistema educativo estatal. Asimismo, para garantizar el bienestar de la comunidad escolar las clases presenciales solamente se llevarán a cabo si el semáforo epidemiológico está en verde y en función de la evolución de la pandemia en cada Estado del país (Secretaría de Educación Pública, 2020).

Esta situación demuestra, que el gobernador de Jalisco no está siguiendo las indicaciones de las autoridades educativas nacionales porque el semáforo epidemiológico en el Estado de Jalisco es naranja. Además, la mejor manera de volver a las aulas es por medio del aprendizaje híbrido coordinado a nivel estatal, porque de no hacerlo los estudiantes se contagiarán en las escuelas, ocasionando un grave problema de salud, lo que conduciría a un grave problema en la educación pública como el que se está viviendo en las escuelas de Estados Unidos (Navarro, 2020).

\section{Conclusiones}

México necesita estar preparado para enfrentar la pandemia del coronavirus por lo que en la Educación en México se tienen que sistematizar las estrategias para la impartición de clases a distancia retomando los proyectos de la UNESCO, UNICEF y SEP. La educación en todo el mundo va a evolucionar por lo que en México se deben adecuar los planes de trabajo y programas de estudio para competir con los estándares internacionales. Es muy importante la utilización de las nuevas tecnologías, pero es necesario tener en cuenta el contexto socioeconómico de las familias para incluir, en el proyecto educativo virtual, a los estudiantes que no tienen acceso a una computadora con internet, de lo contrario la rezago educativo será cada vez más grande.

Uno de los grandes retos en México durante esta pandemia es impartir clases a distancia a más de 34 millones de estudiantes en México porque representan casi un 30\% de la población. La forma más segura de regresar a las clases presenciales es por medio del aprendizaje híbrido coordinado a nivel nacional y estatal. Asimismo, se debe fortalecer el sistema educativo para que no crezca la brecha socioeducativa y garantizar el acceso a tecnologías de educación no solamente en las escuelas sino también en las casas de los alumnos de bajos recursos. 
Por lo tanto, es necesario que, en México, en las escuelas desde educación básica hasta superior, existan programas de préstamos de computadoras. Como se ha mostrado, solamente un 16.4\% de las familias que pertenecen a un estrato socioeconómico bajo tienen conexión a internet, por lo que proporcionar acceso gratuito a dispositivos con internet a los alumnos de bajos recursos permitirá impartir una educación virtual equitativa. Para disminuir las desigualdades y ofrecer una educación inclusiva se deben generar acciones concretas que garanticen educación en las poblaciones excluidas, así como capacitaciones para los docentes de manera que puedan impartir una educación a distancia que motive a los estudiantes en estos tiempos de pandemia.

\section{Referencias}

Andújar Palma, T. (28 abril 2020). “La capacitación digital de los docentes, el desafío de la educación que viene". Agencia EFE. Consultado 23 de julio 2020. URL:

https://www.efe.com/efe/espana/portada/la-capacitacion-digital-de-los-docentes-el-desafioeducacion-que-viene/10010-4233327

Ayuso, M. (2020, abril). "Francesco Tonucci: 'Si el virus cambió todo, la escuela no puede seguir igual'”. La Nación. Consultado 24 de abril 2020, URL:

https://www.lanacion.com.ar/comunidad/francesco-tonucci-si-virus-cambio-todo-escuela$\underline{\text { nid } 2356227}$

Bédard, F., Pelletier, P., Le Clech, C. (2017). “L'apprentissage hybride". Pédagogie univérsitaire. Consultado el 27 de julio 2020. URL:

http://pedagogie.uquebec.ca/le-tableau/lapprentissage-hybride

Campuzano, J. (15 de mayo 2020). "Maestros en cuarentena: retos de la educación y las clases en línea". Milenio. Consultado 23 de julio 2020. URL:

https://www.milenio.com/politica/coronavirus-mexico-educacion-cuarentena-retos-maestros

Desde Casa. Programa de préstamo de computadoras (2020). Gaceta universitaria. Universidad de Guadalajara. Consultado 23 de julio 2020. URL:

http://www.gaceta.udg.mx/desde-casa/ 
Educación Jalisco (2020, abril). “Recrea digital”. Consultado 4 de mayo 2020, URL:

https://recreadigital.jalisco.gob.mx/

Expansión política. (2020, marzo). "Por coronavirus, al menos diez Estados adelantan la suspensión de clases". Expansión política. Consultado el 23 de abril del 2020, URL:

https://politica.expansion.mx/estados/2020/03/17/estados-adelantan-suspension-de-clases Gobierno de México (2020, abril). "Aprende en casa". Consultado 4 de mayo 2020, URL: https://www.aprendeencasa.mx/aprende-en-casa/

Ley General de Educación (30 de septiembre del 2019). Cámara de Diputados del H. Congreso de la Unión. Consultado 22 de junio 2020, URL:

http://www.diputados.gob.mx/LeyesBiblio/pdf/LGE_300919.pdf

Maestras y Maestros en Casa (2020). Gobierno de México. Consultado 23 de junio 2020, URL:

https://segacademcb.cbachilleres.edu.mx/secciones/maestras-maestros-casa.html

Navarro, B. (2020, 23 de noviembre). "La escuela americana sucumbe al virus". La Vanguardia. URL: https://www.lavanguardia.com/internacional/20201123/49598940205/escuelas-cerradas-estadosunidos-coronavirus.html

Peñalosa Castro, E. (16 de julio 2020). “Papel de las tecnologías en la educación superior en la nueva normalidad: una propuesta". Transmisión de la Academia Mexicana de Ciencias (AMC) en Youtube (AMC). URL: https://www.youtube.com/acamexcie

Préstamo de portátiles (10 de junio 2020). UAM Biblioteca. Universidad Autónoma de Madrid. Consultado 23 de julio 2020. URL:

https://biblioguias.uam.es/prestamo/portatilles

Programa de las Naciones Unidas para el Desarrollo (PNUD) (2020). “Desarrollo humano y Covid-19 en México: desafíos para una recuperación sostenible". Consultado el 18 de julio 2020, URL: https://www.mx.undp.org/content/mexico/es/home/library/poverty/desarrollo-humano-y-covid19-en-mexico-.html

Ramírez León, R. H. (2016). "Los retos que impone la educación a distancia en México" en Revista Iberoamericana de Producción Académica y Gestión Educativa. №. 6, julio- diciembre. 
Ramírez, V. (2020, 2 de diciembre). “Alfaro plantea regreso a clases presenciales en Jalisco el 25 de enero". El Sol de México. URL:

https://www.elsoldemexico.com.mx/republica/sociedad/alfaro-plantea-regreso-a-clasespresenciales-en-jalisco-el-25-de-enero-2021-covid-19-6085981.html

Secretaría de Educación Pública (SEP). (22 de julio de 2020). “Boletín No. 196. Convivirán en el futuro, modelo de educación presencial y a distancia: Esteban Moctezuma Barragán”. Consultado el 27 de julio 2020. URL:

https://www.gob.mx/sep/articulos/boletin-no-196-conviviran-en-el-futuro-modelo-de-educacionpresencial-y-a-distancia-esteban-moctezuma-barragan?idiom=es

Servín, A. (4 de julio 2020). "Hacia una educación híbrida ante la nueva normalidad”. El Economista. Consultado el 27 de julio 2020. URL:

https://www.eleconomista.com.mx/arteseideas/Hacia-una-educacion-hibrida-ante-la-nuevanormalidad-20200704-0022.html

Silió, E. (2020, abril). “Castells prevé un próximo curso universitario con turnos rotatorios en aulas desinfectadas". El País. Consultado 24 de abril 2020, URL:

https://elpais.com/sociedad/2020-04-23/castells-preve-un-proximo-curso-universitario-con-turnosrotatorios-en-aulas-desinfectadas.html

UNESCO (2002). Aprendizajes abiertos y a distancia. Montevideo: Ediciones Trilce.

UNESCO (2020a, abril). “Coalición mundial para la educación Covid-19”. Consultado 24 de abril 2020, URL:

https://es.unesco.org/covid19/globaleducationcoalition

UNESCO (2020b, abril). “La educación en América Latina y el Caribe ante el Covid-19". Consultado 4 de mayo 2020, URL:

https://es.unesco.org/fieldoffice/santiago/covid-19-education-alc

UNICEF (2020, abril). “\#Aprendo en casa. Juega y aprende con tus hijos e hijas en tiempos de Covid19". Consultado 4 de mayo 2020, URL:

https://www.unicef.org/mexico/aprendoencasa-\%C3\%BAnete-al-reto 
Vázquez, A. (27 de abril 2020). Lanza UdeG clases virtuales “Desde casa”. El Occidental. Consultado 23 de julio 2020. URL:

https://www.eloccidental.com.mx/local/lanza-udeg-clases-virtuales-desde-casa-coronavirus-covid19-pandemia-oms-fase-3-mexico-ricardo-villanueva-lomeli-prestamo-computadoras-semsudgvirtual-5159262.html. 